\title{
On the Maximum Coverage Area of Wireless Networked Control Systems with Maximum Cost-Efficiency under Convergence Constraint
}

\author{
Deniz Kilinc, Student Member, IEEE, Mustafa Ozger, Student Member, IEEE, \\ and Ozgur B. Akan, Senior Member, IEEE
}

\begin{abstract}
The integration of wireless communication and control systems revealed wireless networked control systems (WNCSs). One fundamental problem in WNCSs is to have a wide coverage area. For the first time in the literature, we address this problem and we obtain the maximum coverage area by solving an optimization problem. In this technical note, we consider a WNCS where the output sensor measurements are transmitted over separate heterogeneous multi-hop wireless ad-hoc subnetworks. The observation process is divided into $N$ parts and the system state is estimated using the Kalman filter. We present the critical arrival probability for a sensor measurement packet such that if the packet arrival probability is larger than the critical value, it is guaranteed that the estimator of the WNCS converges. We derive the maximum total coverage area of the heterogeneous wireless subnetworks having maximum cost-efficiency under the constraint of the convergence of the WNCS estimator.
\end{abstract}

Index Terms-Wireless networked control systems, multi-hop wireless networks, coverage area, estimator convergence, Kalman filtering.

\section{INTRODUCTION}

Recent developments on micro sensor integrated systems have enabled combination of communication and control systems. This integration revealed networked control systems (NCSs) where the communication system enables the sensor observation delivery [1], [2]. The control system components such as sensors, actuators and plants with wireless communication capabilities constitute a wireless networked control system (WNCS). The observations of the sensors deployed over a wide area are fed to the WNCS through a wireless network. The WNCSs have a wide application area such as smart grid, automatic management and navigation systems [3].

For the WNCS applications requiring large coverage areas, e.g., space and terrestrial exploration, navigation systems, the maximum achievable area of the wireless network which ensures the convergence of the WNCS estimator is crucial. To the best of our knowledge, no attempt has yet been made to find the maximum coverage area of the wireless network under the convergence constraint. For the first time in the literature, we address this problem and obtain the solution by solving an optimization problem in [4]. Although in [5], [6], [7], the authors study the maximum coverage area for

The authors are with the Next-generation and Wireless Communications Laboratory, Department of Electrical and Electronics Engineering, Koc University, Istanbul, 34450, Turkey (e-mail: \{dkilinc, mozger, akan\}@ku.edu.tr)

This work was supported by the Turkish Scientific and Technical Research Council under grant \#110E249. wireless networks, they do not consider the convergence of a WNCS estimator which utilizes wireless networks. We find the maximum coverage area of a wireless network having maximum cost-efficiency by considering the convergence of the WNCS estimator.

In our scenario, wireless sensor nodes are employed to observe the system behavior. We consider that the sensor measurements are transmitted to the controller over multi-hop wireless ad-hoc networks. As an extension of our previous study in [4], we employ a heterogeneous multi-hop wireless ad-hoc network model as a generalization of homogeneous networks. In these networks, also known as cognitive radio sensor networks [8], [9], measurement packets are conveyed to the WNCS estimator by utilizing available channels opportunistically. These packets may be lost due to the unreliable wireless channel characteristics caused by the noise, collision, and congestion. Since the WNCSs rely on the observations of the sensors to estimate the state of the system, any loss of the sensor measurements degrades the stability of the WNCS.

We use the Kalman filter for the state estimation of the system. The Kalman filtering is a well studied technique in control theory [10], [11]. In the classical sense, the Kalman filter uses all the observation data provided by the sensors for the state estimation. However, for the WNCSs, the observations may be lost due to wireless channel conditions. In [10], the Kalman filter is studied when the observations are intermittent; nevertheless, the authors do not consider statistical convergence behavior. In [11], the authors investigate the state estimation process, in which the sensor measurements are received or lost completely in a stochastic manner, and they show that if the probability of arrival of an observation is above a threshold, the expectation of the state estimation error covariance is bounded. In [12], the authors consider two sensors, and the measurement of each sensor is independently received or lost by the Kalman filter. Furthermore, in [13], the $H_{\infty}$ filtering problem is studied for a class of discrete-time networked nonlinear systems with random delays and packet losses; and in [14], the distributed fuzzy filters are designed so that the filtering error dynamic system to be mean-square stable in spite of time-varying delays and multiple probabilistic packet losses. However, none of these works address the maximum coverage area problem of a WNCS network under the convergence constraint.

We consider the general case of the system presented in [12]. The observation process is divided into $N$ parts and each part is independently and randomly received or lost 
by the Kalman filter. Thus, we consider $N$ separate multihop wireless ad-hoc subnetworks for our scenario and each subnetwork includes one sensor node. Based on the derivations presented in [12], we derive the critical arrival probability for the measurement of each sensor such that if the arrival probability of a sensor measurement is larger than the critical value, it is guaranteed that the estimator of the WNCS is convergent so that the WNCS is stable. In this technical note, we refer the stability of the WNCS as the convergence of the WNCS estimator. Then, we show that there exists a critical hop-diameter of a subnetwork such that if the hop-diameter of the subnetwork is less than the critical hop-diameter, the WNCS is stable where the maximum hop number of the shortest paths between any two node pairs in the network is the hop-diameter. Furthermore, based on the solution of an optimization problem, we find both the optimum hopdiameter and the maximum coverage area of the multi-hop wireless ad-hoc network with maximum cost-efficiency under the constraint of the convergence of the WNCS estimator.

\section{Kalman Filtering with Partial Observation LOSSES}

In a WNCS, the Kalman filter gathers sensor measurements from distinct sensors and each sensor node encodes its own observation into a single packet. However, some of the packets might be lost during the wireless data transmission. In [12], the authors present a state estimation process with partial observation losses considering that the observation process is divided into two parts which are transmitted over different wireless channels by two different sensor nodes. In this section, we present a general state estimation process, i.e., the observation process is divided into $N$ parts, with partial observation losses using the Kalman filter. In other words, the Kalman filter uses the output observations of $N$ independent sensors.

We consider a general multiple-input multiple-output (MIMO) discrete time linear time-invariant system which is described by the following system equations

$$
\begin{gathered}
\mathbf{x}_{t+1}=A \mathbf{x}_{t}+\mathbf{w}_{t}, \\
{\left[\begin{array}{c}
\mathbf{y}_{1, t} \\
\vdots \\
\mathbf{y}_{N, t}
\end{array}\right]=\left[\begin{array}{c}
C_{1} \\
\vdots \\
C_{N}
\end{array}\right] \mathbf{x}_{t}+\left[\begin{array}{c}
\mathbf{v}_{1, t} \\
\vdots \\
\mathbf{v}_{N, t}
\end{array}\right]}
\end{gathered}
$$

where $\mathbf{x}_{t} \in \mathcal{R}^{n}$ is the system state vector, $\mathbf{w}_{t} \in \mathcal{R}^{n}$ is the system disturbance vector, $A \in \mathcal{R}^{n \times n}$ is the system matrix, $\mathbf{y}_{i, t} \in \mathcal{R}^{m_{i}}$, is sensor measurement output vector, $\mathbf{v}_{i, t} \in \mathcal{R}^{m_{i}}$ is the measurement noise vector, and $C_{i} \in \mathcal{R}^{m_{i} \times n}$ is the output matrice for $i=1,2, \ldots, N$ and the subscript $t$ indicates the time index. Also note that the boldface symbols in this technical note represent vectors. We define $\mathbf{y}_{t}=$ $\left[\begin{array}{l}\mathbf{y}_{1, t}, \\ \mathbf{y}_{2, t}, \ldots, \mathbf{y}_{N, t}\end{array}\right]^{\mathrm{T}}, \mathbf{v}_{t}=\left[\mathbf{v}_{1, t}, \mathbf{v}_{2, t}, \ldots, \mathbf{v}_{N, t}\right]^{\mathrm{T}}$, and $C=\left[C_{1}, C_{2}, \ldots, C_{N}\right]^{\mathrm{T}}$. Both $\mathbf{w}_{t}$ and $\mathbf{v}_{t}$ are assumed to be Gaussian random vectors with zero mean and their covariance matrices are $Q \geq 0$ and $R>0$, respectively. $R$ is a $N \mathrm{x} N$ matrix having elements as $R_{i j}=\mathrm{E}\left[\mathbf{v}_{i, t} \mathbf{v}_{j, t}^{\prime}\right]$. Furthermore, we assume that the system $(A, C)$ is observable; hence, the Kalman filter converges without sensor measurement losses. (a)

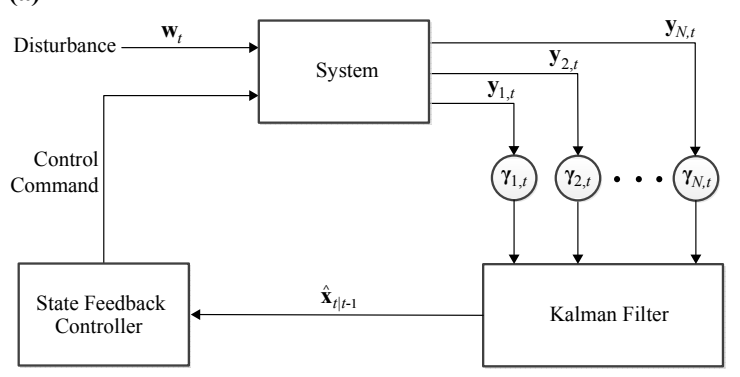

(b)

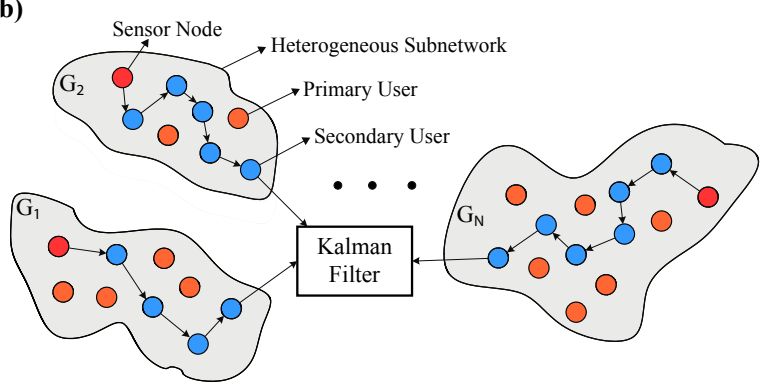

Fig. 1. (a) The block diagram of the WCSN. (b) The model of the heterogeneous multi-hop wireless ad-hoc subnetworks.

The sensor measurement packets $\mathbf{y}_{1, t}, \mathbf{y}_{2, t}, \ldots, \mathbf{y}_{N, t}$ are encoded independently and transmitted over different multihop wireless ad-hoc subnetworks. We use random variable $\gamma_{i, t}$ which indicates whether the measurement packet of $i^{\text {th }}$ sensor, $\mathbf{y}_{i, t}$, is correctly received during a given sample period. We assume $\gamma_{i, t}$ for $i=1,2, \ldots, N$ are independent Bernoulli random variables with $\operatorname{Pr}\left\{\gamma_{i, t}=1\right\}=\lambda_{i}$ and $\operatorname{Pr}\left\{\gamma_{i, t}=0\right\}=1-\lambda_{i}$. That is, if $\gamma_{i, t}=1$, then the measurement packet $\mathbf{y}_{i, t}$ is correctly received; otherwise, the packet is lost during the wireless data transmission. $\lambda_{i}$ depends on the channel gains, the network resource allocation, the network traffic, and the number of hops taken by a packet to reach the Kalman filter. In this technical note, we are only interested in the successful transmission probability of a packet between two nodes and we do not consider the effects of modulation, decoding and encoding processes of the information on the successful transmission probability of a packet.

The block diagram of the WNCS for our scenario is shown in Fig. 1(a). Note that the observation process is stochastic due to the random measurement losses during the packet transmission process. Furthermore, since we assume that $\gamma_{i, t}$ and $\gamma_{j, t^{\prime}}$ for $i \neq j$ are independent for every $t$ and $t^{\prime}$, the sensor measurement packets $\mathbf{y}_{i, t}$ for $i=1,2, \ldots, N$ can be independently lost or received. Therefore, the loss of a measurement packet is equivalent to the reception of a measurement having an infinite noise variance. Then, for the measurement noise vectors $\mathbf{v}_{i, t}$, we define the following conditional probability distribution function $f_{\mathbf{v} \mid \gamma}\left(\mathbf{v}_{i, t} \mid \gamma_{i, t}\right) \sim \mathcal{N}\left(0, R_{i i}\right)$ if $\gamma_{i, t}=1$ and $f_{\mathbf{v} \mid \gamma}\left(\mathbf{v}_{i, t} \mid \gamma_{i, t}\right) \sim \mathcal{N}\left(0, \sigma_{i}^{2} I\right)$ if $\gamma_{i, t}=0$. Then, we take the limit as $\sigma_{i}^{2} \rightarrow \infty$ to derive the Kalman equations for random partial losses.

In [11], the authors investigate the state estimation pro- 
cess, in which the sensor measurement packet is received or lost completely, and they show the existence of a critical packet arrival probability $\lambda^{c}$ such that $\mathrm{E}\left[P_{t+1 \mid t}\right]$ is bounded if $\lambda>\lambda^{c}$ and $\mathrm{E}\left[P_{t+1 \mid t}\right]$ becomes infinite as $t \rightarrow \infty$ if $\lambda<\lambda^{c}$. For the general case, based on the derivations and results given in [12], if $(A, Q)$ is controllable and $(A, C)$ is observable, for a fixed set of $\left(\lambda_{1}, \lambda_{2}, \ldots, \lambda_{i-1}, \lambda_{i+1}, \ldots \lambda_{N}\right)$, if $\lambda_{i} \geq \lambda_{i}^{c}$, the state estimation error covariance is bounded and estimator converges so that the WNCS is stable. If the output matrices $C_{1}, C_{2}, \ldots, C_{N}$ are square and invertible $A$ has a single unstable eigenvalue, the upper and lower bounds for $\lim _{t \rightarrow \infty} \mathrm{E}\left[P_{t+1 \mid t}\right]$ coincide and the critical packet arrival probability of the measurement packet of the $i^{\text {th }}$ sensor becomes

$$
\begin{aligned}
& \lambda_{i}^{c}=\max \{0, \\
& \left.1-\frac{1}{\alpha^{2}\left(1-\lambda_{1}\right) \ldots\left(1-\lambda_{i-1}\right)\left(1-\lambda_{i+1}\right) \ldots\left(1-\lambda_{N}\right)}\right\}
\end{aligned}
$$

where $\alpha=\max _{i}\left|\sigma_{i}\right|$ and $\sigma_{i}$ is the $i^{\text {th }}$ eigenvalue of $A$ [12]. We discuss the appropriate selection of the set of $\left(\lambda_{1}, \lambda_{2}, \ldots, \lambda_{N}\right)$ in Section IV for a cost-efficient WNCS with the maximum coverage area under convergence constraint.

\section{Multi-Hop Wireless Ad-Hoc Network Model AND CONNECTIVITY}

For the WNCS, we employ multi-hop wireless ad-hoc networks. The first advantage of multi-hop wireless ad-hoc networks is that they can be employed in a fast and easy way, which is the reason why they are named "ad-hoc networks" [15]. The second advantage of this network model is that very large areas can be covered by means of the multi-hop property. However, since the wireless channels are unreliable, as the number of hops increases during the packet transmission, the packet arrival probability decreases.

In this technical note, we consider a heterogeneous multihop wireless ad-hoc network model in which cognitive radio ad-hoc networks (secondary networks) coexist with licensed networks (primary networks). In such a heterogeneous network, the secondary network nodes, i.e., Secondary Users (SUs), cannot communicate with the primary network nodes, i.e., Primary Users (PUs). Cognitive radio ad-hoc networks enable the unlicensed secondary users to utilize the spectrum holes unoccupied by the licensed PUs so that the limited spectrum resource is more efficiently used [9]. For the analysis presented in this part, we assume that the sensor measurements are transmitted to the Kalman filter over the cognitive radio multi-hop wireless ad-hoc subnetworks.

We assume that the PUs (SUs) are distributed according to two dimensional homogeneous Poisson point process having a density $\rho_{p}\left(\rho_{s}\right)$. We consider that the transmission ranges of all primary (secondary) network nodes are the same and denoted by $r_{p}\left(r_{s}\right)$. The connectivity of cognitive radio networks is more troublesome than the connectivity of homogeneous networks. That is, the secondary cognitive radio network is connected if each node pair $\mathrm{SU}_{i}$ and $\mathrm{SU}_{j}$ in the cognitive radio network satisfies the following conditions: 1) The distance between $\mathrm{SU}_{i}$ and $\mathrm{SU}_{j}, r$, satisfies $r \leq r_{s}$ condition. 2) Both $\mathrm{SU}_{i}$ and $\mathrm{SU}_{j}$ are outside the transmission range $r_{p}$ of every active sender in the primary network. 3) There is no active primary network receiver in the transmission range $r_{s}$ of $\mathrm{SU}_{i}$ and $\mathrm{SU}_{j}$. In Fig. 1(b), the heterogeneous multi-hop wireless ad-hoc network model used in this technical note is shown where $G_{i}$ denotes the $i^{\text {th }}$ cognitive radio subnetwork including the $i^{\text {th }}$ sensor node.

In [15], the authors show that there exists a critical node density $\rho_{s}^{*}$ such that if the node density of the secondary network is larger than $\rho_{s}^{*}$, the secondary network percolates at all time, i.e., there exists always an infinite connected component in the secondary network under the time-varying spectrum availability. To guarantee the connectivity of the secondary network, we use the upper bound of the critical node density which is given by [15]

$$
\rho_{s}^{*}=\frac{5}{r_{s}^{2}} \ln \left[1-\sqrt{\left(1-(\sqrt{6} / 3)^{\Lambda}\right) e^{\left(\left|R_{e}\right|+\left|R_{e}^{\prime}\right|\right) \Pi_{1} \rho_{p}}}\right]^{-1}
$$

where $\Lambda=(4 L d+2 d+1) \times(4 L d+d)+(4 L d+d+1) \times(4 L d+$ $2 d)-1,\left|R_{e}\right|=\left(2+2\left\lceil r_{p} / d\right\rceil\right) \times\left(1+2\left\lceil r_{p} / d\right\rceil\right) \times d^{2},\left|R_{e}^{\prime}\right|=$ $\left(2+2\left\lceil r_{s} / d\right\rceil\right) \times\left(1+2\left\lceil r_{s} / d\right\rceil\right) \times d^{2}, L=\left\lceil\max \left\{r_{p}, r_{s}\right\} / d\right\rceil$, $d=r_{s} / \sqrt{5}$. We assume that each PU sender is associated with an independent and identically distributed (i.i.d.) alternating renewal process ${ }^{1}$, denoted by $S_{p}(t)$, which alternates between two states: the ON state, during which the licensed channel is used by the PU, i.e., the PU is active; and the OFF state, during which the licensed channel is not used by the PU, i.e., the PU is inactive. $\Pi_{1}$ in (3) is defined as the probability that the licensed channel is used by a PU, i.e., $\Pi_{1}=\operatorname{Pr}\left\{S_{p}(t)=1\right\}$.

\section{Maximum Coverage Area Analysis}

In a multi-hop network, we can increase the coverage area by increasing the number of nodes in the network. However, if the coverage is enlarged with an increase in the number of nodes, the number of hops during the packet transmission between two distant nodes rises. Because of the unreliable wireless channels, an increase in the number of hops during the transmission decreases the successful packet arrival probability, and the estimator of the WNCS might diverge and WNCS become unstable as discussed in Section II. Thus, for a stable WNCS, the hop-diameter of the network, denoted by $d_{i}$, becomes a critical parameter.

The successful packet transmission probability between two nodes, which are within the transmission range of each other, is assumed to be constant and the same for each transmission process in the network and it is denoted by $\beta$. Therefore, the probability that the $i^{\text {th }}$ sensor measurement is correctly received by the Kalman filter, i.e., $\lambda_{i}$, can be expressed as $\lambda_{i}=\beta^{M_{i}}$ for $i=1,2, \ldots, N$ where $M_{i}$ is the number of hops taken by the packet transmitted by the $i^{\text {th }}$ sensor until it reaches the Kalman filter. $M_{i}$ depends on the routing protocol, network topology, and number of nodes. Here, we assume that

\footnotetext{
${ }^{1}$ An alternating renewal process is a two-state process which alternates between an on-state and an off-state after a random sojourn time, where the on-times and the off-times are independent random variables.
} 


$$
\begin{aligned}
& \underset{\lambda_{1}, \ldots, \lambda_{N}}{\operatorname{maximize}} f\left(\lambda_{1}, \ldots, \lambda_{N}\right)=\lambda_{1} m_{1}\left(\lambda_{1}\right)+\lambda_{2} m_{2}\left(\lambda_{2}\right)+\cdots+\lambda_{N} m_{N}\left(\lambda_{N}\right) \\
& \text { subject to } \lambda_{i} \geq \max \left\{0,1-\frac{1}{\alpha^{2}\left(1-\lambda_{1}\right) \ldots\left(1-\lambda_{i-1}\right)\left(1-\lambda_{i+1}\right) \ldots\left(1-\lambda_{N}\right)}\right\} \quad \text { for } i=1,2, \ldots, N
\end{aligned}
$$

the routing protocol is smart enough so that it can find a single shortest route from the sensor to the Kalman filter [16].

Let the critical packet arrival probability of the $i^{\text {th }}$ sensor measurement be $\lambda_{i}^{c}$. Based on the definition of the hopdiameter of a network, the maximum number of hop taken by a measurement packet until it reaches the Kalman filter is less than or equal to the hop-diameter of the subnetwork. Then, using $\lambda_{i}=\beta^{M_{i}}$, the critical-hop diameter of $i^{\text {th }}$ subnetwork is $d_{i}^{c}=\left\lfloor\ln \left(\lambda_{i}^{c}\right) / \ln (\beta)\right\rfloor$. That is, if the hop-diameter of the subnetwork $G_{i}$ satisfies $d_{i} \leq d_{i}^{c}$ condition, it is guaranteed that the arrival probability of the packet transmitted by $i^{\text {th }}$ sensor is larger than the critical arrival probability; hence, the estimator of the WNCS converges. However, if $d_{i}>d_{i}^{c}$, the convergence of the estimator is not guaranteed. Since the hop-diameter depends on several factors such as topology, network size, node locations, sensor communication range, and node density, it is difficult to find an upper bound for the maximum number of nodes which ensures a given hop-diameter. Therefore, to guarantee the convergence of the estimator, one can use lower bound for the maximum number of nodes in a subnetwork given as $m_{i}\left(\lambda_{i}^{c}\right)=d_{i}^{c}+1$ where $m_{i}\left(\lambda_{i}^{c}\right)$ denotes the number of nodes which guarantees that the packet arrival probability is greater than the critical value and the proof is straightforward.

If we consider only the convergence criterion, for a given set of $\left(\lambda_{1}, \lambda_{2}, \ldots, \lambda_{i-1}, \lambda_{i+1}, \ldots \lambda_{N}\right)$, as $\lambda_{i}^{c} \rightarrow 0, m_{i}\left(\lambda_{i}^{c}\right) \rightarrow$ $\infty$, and hence the total coverage area of the $i^{\text {th }}$ subnetwork becomes infinite. Indeed, it is irrational and cost-inefficient to place infinitely many nodes in a subnetwork including a sensor node whose critical packet arrival probability is 0 . That is, a decrease in $\lambda_{i}^{c}$ decreases the importance of the subnetwork $G_{i}$, and when $\lambda_{i}^{c}=0$, the measurements of the $i^{\text {th }}$ sensor in $G_{i}$ become unnecessary for the convergence of the WNCS estimator. Thus, the cost-efficiency of the multi-hop wireless network should also be considered because the selection of a set of packet arrival probabilities of the sensor measurements affects the cost-efficiency.

Since when $\lambda_{i} \rightarrow 0, m_{i}\left(\lambda_{i}^{c}\right) \rightarrow \infty$ and $\lambda_{i} m_{i}\left(\lambda_{i}\right) \rightarrow 0$, we can use $\lambda_{i} m_{i}\left(\lambda_{i}\right)$ as the efficiency of the $i^{\text {th }}$ subnetwork. Therefore, to find a cost-efficient multi-hop wireless ad-hoc network, we define a cost-efficiency function as follows

$$
f\left(\lambda_{1}, \ldots, \lambda_{N}\right)=\lambda_{1} m_{1}\left(\lambda_{1}\right)+\cdots+\lambda_{N} m_{N}\left(\lambda_{N}\right)
$$

where $m_{i}\left(\lambda_{i}\right)$ is the number of nodes in the subnetwork $G_{i}$ which guarantees that the packet arrival probability is bounded below by $\lambda_{i}$ and it is given by $m_{i}\left(\lambda_{i}\right)=\left\lfloor\ln \left(\lambda_{i}\right) / \ln (\beta)\right\rfloor+$ 1. Note that the cost-efficiency function is the weighted sum of the number of nodes in the subnetworks. Using (2), the set of $\left(\lambda_{1}, \lambda_{2}, \ldots, \lambda_{N}\right)$ which both maximizes $f\left(\lambda_{1}, \ldots, \lambda_{N}\right)$ and ensures the convergence of the WNCS estimator can be found by solving the optimization problem in (5) at the top of this page. Note that, the convergence condition of a WNCS estimator is $\lambda_{i} \geq \lambda_{i}^{c}$ for $i=1,2, \ldots, N$ where $\lambda_{i}^{c}$ is given in (2) as discussed in Section II.

We numerically evaluated the objective function in (5) to obtain a pattern for the solution. Since there is a constraint of the maximization problem, the maximum point changes according to the constraint. That is, if the constraint functions for $\lambda_{i}$ for $i=1,2, \ldots, N$ are small enough, the maximum point is equal to the global maximum of the objective function and the global maximum point is given by $e^{-\ln \left(\lambda_{i}\right)-1}$, which is an analytical result. However, if the constraint functions are larger than a certain value, the maximum is found by considering both the objective and constraint functions. For this second case, we found that the maximum value of the objective function in (5) can be approximately given by $1-\alpha^{-2 / N}$ according to the numerical evaluations of the objective function. Therefore, the solution of (5) can be approximated as

$$
\lambda_{i}^{\mathrm{opt}} \approx \max \left\{e^{-\ln (\beta)-1}, 1-\alpha^{-2 / N}\right\}
$$

for $i=1,2, \ldots, N$, where $\left(\lambda_{1}^{\mathrm{opt}}, \lambda_{2}^{\mathrm{opt}}, \ldots, \lambda_{N}^{\mathrm{opt}}\right)$ denotes the optimum convergent set having the maximum cost-efficiency. Note that, since we used an approximation for the second case of the optimization problem in (5), the solution given in (6) is an approximate solution obtained using an ad hoc method. The results show that the solution given in (6) accurately satisfies the convergence constraint of the WNCS estimator and maximizes the cost-efficiency.

Using the optimum set of packet arrival probabilities given in (6), the optimum hop-diameter of the $i^{\text {th }}$ subnetwork having the maximum cost-efficiency is given by

$$
d_{i}^{\text {opt }}=\left\lfloor\frac{\ln \left(\max \left\{e^{-\ln (\beta)-1}, 1-\alpha^{-2 / N}\right\}\right)}{\ln (\beta)}\right\rfloor .
$$

Furthermore, to guarantee the convergence of the WNCS estimator, we use the lower bound for the maximum number of nodes in $G_{i}$, denoted by $m_{i}\left(\lambda_{i}^{\text {opt }}\right)$, and it is

$$
m_{i}\left(\lambda_{i}^{\mathrm{opt}}\right)=\left\lfloor\frac{\ln \left(\max \left\{e^{-\ln (\beta)-1}, 1-\alpha^{-2 / N}\right\}\right)}{\ln (\beta)}\right\rfloor+1
$$

for $i=1,2, \ldots, N$. Based on the number of nodes in each subnetwork having maximum cost-efficiency under the convergence constraint, we can derive the maximum coverage of the WNCS. Note also that, (6), (7), and (8) are independent of $i$ which is an expected result because we consider each subnetwork identical. In our future work, we aim to consider subnetworks having different parameters to make the scenario more realistic.

To find the coverage area of the subnetworks for the number of nodes given in (8), we consider the connectivity of the subnetworks. Thus, to have the maximum coverage area for a given number of nodes, we assume that the node density of the heterogeneous multi-hop wireless ad-hoc network is the 
same as the critical node density $\rho_{s}^{*}$ in (3). Then, for a stable WNCS, the maximum coverage area of the subnetwork $G_{i}$, which has the maximum cost-efficiency, is given by $S_{i}^{h t}=$ $m_{i}\left(\lambda_{i}^{\mathrm{opt}}\right) / \rho_{s}^{*}$. Since the number of nodes found in (8) is the same for each subnetwork, the maximum total coverage area of the heterogeneous subnetworks is given by

$$
S_{\mathrm{T}}^{h t}=\frac{N m_{i}\left(\lambda_{i}^{\mathrm{opt}}\right) r_{s}^{2}}{5 \ln \left[1-\sqrt{\left(1-(\sqrt{6} / 3)^{\Lambda}\right) e^{\left(\left|R_{e}\right|+\left|R_{e}^{\prime}\right|\right) \Pi_{1} \rho_{p}}}\right]^{-1}} .
$$

\section{Numerical ANalysis}

In this section, we present the numerical analyses of both the optimum hop-diameter $d_{i}^{\mathrm{opt}}$ and the maximum total coverage area $S_{\mathrm{T}}^{h t}$ of the heterogeneous subnetworks with respect to several system and network parameters. We assume that the observation model matrices $C_{1}, C_{2}, \ldots, C_{N}$ are square and invertible [11], [12]. Note that, the observation model matrices map the true state space into the observed space and in general, their dimensions are $m_{i} \times n$ for $i=1,2, \ldots, N$. If $C_{i}$ is a square matrix, the true state space and the observed space have the same dimensions, which is a quite reasonable assumption. The numerical evaluations are conducted using MATLAB.

\section{A. Optimum Hop-Diameter of Subnetworks}

In the first part of the numerical analyses, we present the variation of the optimum hop-diameter of subnetworks, $d_{i}^{\text {opt }}$, given in (7) with respect to the number of sensor nodes, $N$, the successful packet transmission probability between two nodes, $\beta$, and the eigenvalue of $A$ having the maximum magnitude, $\alpha$.

In Fig. 2(a), $d_{i}^{\text {opt }}$ with respect to the number of sensor nodes $N$ employed for the WNCS with different $\beta$ values is shown. $d_{i}^{\text {opt }}$ increases with an increase in $\beta$ which is an expected result. Note that $0 \leq \beta \leq 1$ and as $\beta \rightarrow 1$, $\ln (\beta) \rightarrow 0$, also the numerator in (7) is negative; hence, an increase in $\beta$ causes an increase in $d_{i}^{\text {opt }}$. As seen in Fig. 2(a), $d_{i}^{\text {opt }}$ increases up to $N=5$, then it becomes constant. If $N>-2 \ln (\alpha) / \ln \left(1-e^{-\ln (\beta)-1}\right)$, then $\max \left\{e^{-\ln (\beta)-1}, 1-\right.$ $\left.\alpha^{-2 / N}\right\}=e^{-\ln (\beta)-1}$, and hence $d_{i}^{\text {opt }}$ depends only on $\beta$. On the other hand, if $N<-2 \ln (\alpha) / \ln \left(1-e^{-\ln (\beta)-1}\right)$, then $\max \left\{e^{-\ln (\beta)-1}, 1-\alpha^{-2 / N}\right\}=1-\alpha^{-2 / N}$; thus, $d_{i}^{\text {opt }}$ depends on $\alpha$ and $N$, i.e., $d_{i}^{\text {opt }}=\ln \left(1-\alpha^{-2 / N}\right) / \ln (\beta)$. Obviously, $d_{i}^{\text {opt }}$ decreases with an increase in $\alpha$. For a fixed $\beta=0.9$, the results seen in Fig. 2(b) show that $d_{i}^{\text {opt }}$ decreases with an increase in $\alpha$, which supports our inferences. It is also seen that $d_{i}^{\text {opt }}$ can be increased with an increase in $N$.

\section{B. Maximum Total Coverage Area of Heterogeneous Network}

In this part, we consider the maximum total coverage area of the heterogeneous multi-hop wireless ad-hoc network model. We present the effect of $r_{s}, r_{p}, N, \beta, \alpha$ on the maximum total coverage area of the heterogeneous subnetworks, $S_{h t}^{\mathrm{T}}$, given in (9). For the numerical analysis presented in this part, we consider that the secondary cognitive radio subnetwork $G_{i}$ is connected. Furthermore, we set $\rho_{p}=0.01$ nodes $/ \mathrm{m}^{2}$.
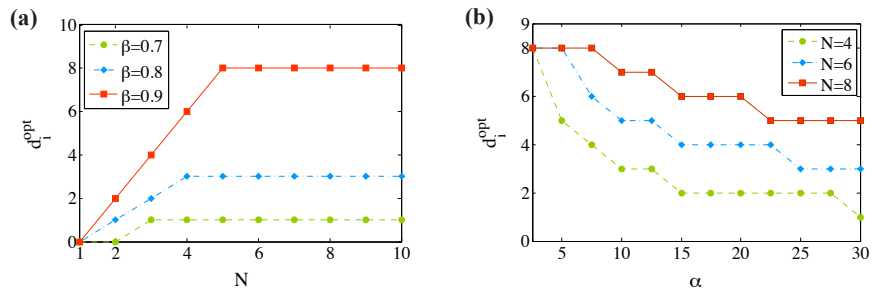

Fig. 2. $\quad d_{i}^{\text {opt }}$ (a) with respect to $N$ for different $\beta$ values and (b) with respect to $\alpha$ for different $N$ values.
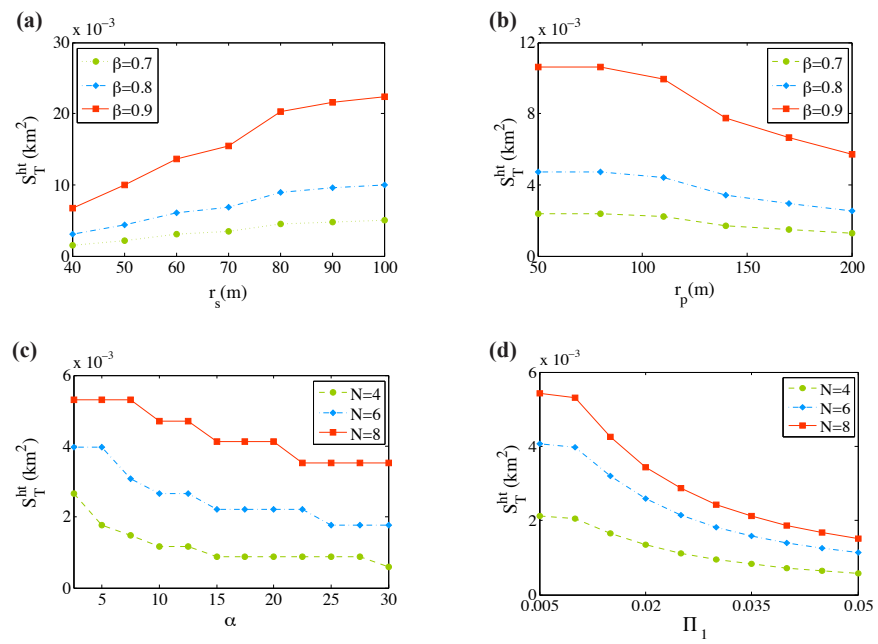

Fig. 3. $S_{\mathrm{T}}^{h t}$ with respect to (a) $r_{s}$ for different $\beta$ values, (b) $r_{p}$ for different $\beta$ values, (c) $\alpha$ for different $N$ values, and (d) $\Pi_{1}$ for different $N$ values.

In Fig. 3(a), for $r_{p}=100 \mathrm{~m}, \Pi_{1}=0.01, N=15$ and $\alpha=$ 4.0 , the variation of the maximum total coverage area $S_{\mathrm{T}}^{h t}$ with respect to the transmission range of SUs, $r_{s}$, is illustrated for different $\beta$ values. According to the results, $S_{\mathrm{T}}^{h t}$ increases with an increase in $r_{s}$ which is an expected result. In addition, the results show that an increase in $\beta$ enlarges the total coverage area of the subnetworks. From Section V-A, $d_{i}^{\text {opt }}$ increases with an increase in $\beta$, and $m_{i}\left(\lambda_{i}^{\mathrm{opt}}\right)=d_{i}^{\mathrm{opt}}+1$. Thus, from (9), it is obvious that $S_{\mathrm{T}}^{h t}$ increases with an increase in $d_{i}^{\text {opt }}$.

In Fig. 3(b), for constant $r_{s}=50 \mathrm{~m}, \Pi_{1}=0.01, N=15$ and $\alpha=4.0$, the variation of the maximum total coverage area $S_{\mathrm{T}}^{h t}$ with respect to the transmission range of PUs, $r_{p}$, is illustrated for different $\beta$ values. According to the results, $S_{\mathrm{T}}^{h t}$ decreases with an increase in $r_{p}$. For connectivity of the secondary network, the PUs are required to be outside the transmission range of SUs as explained in Section III-B. Thus, an increase in the transmission range of PUs decreases the number of connected nodes in the secondary network, which decreases the maximum total coverage area of the secondary subnetworks. In addition, the results show that an increase in $\beta$ can significantly increase the total coverage area of the subnetworks.

The effect of $\alpha$, i.e., the eigenvalue of the system matrix $A$ having the maximum magnitude, on the maximum total coverage area of the multi-hop wireless subnetworks $S^{\mathrm{T}}$ is shown in Fig. 3(c) for different $N$ values. Here, we set $r_{s}=50 \mathrm{~m}, r_{p}=100 \mathrm{~m}, \Pi_{1}=0.01$, and $\beta=0.9$. According to the results, an increase in $\alpha$, causes a reduction in the 
maximum total coverage area $S_{\mathrm{T}}^{h t}$. For a given $N$, an increase in $\alpha$ decreases the optimum hop-diameter. Therefore, since $m_{i}\left(\lambda_{i}^{\mathrm{opt}}\right)=d_{i}^{\mathrm{opt}}+1$, an increase in $\alpha$ also decreases $S_{\mathrm{T}}^{h t}$, which can be seen in (9). Moreover, $S_{\mathrm{T}}^{h t}$ increases with an increase in $N$ because of $S_{\mathrm{T}}^{h t}=N S_{i}^{h t}$. Modifying the system matrix $A$ results in a change in $\alpha$. For example in [17], the authors consider the problem of minimizing the largest eigenvalue of a matrix. Therefore, this technique can be used to change the largest eigenvalue of the system matrix A.

In Fig. 3(d), the effect of $\Pi_{1}$, on the maximum total coverage area of the multi-hop wireless subnetworks $S^{\mathrm{T}}$ for different $N$ values is demonstrated. For this analysis, we set $r_{s}=50 \mathrm{~m}, r_{p}=50 \mathrm{~m}, \alpha=4.0$, and $\beta=0.9$. According to the results, an increase in $\Pi_{1}$, causes a reduction in the maximum total coverage area $S_{\mathrm{T}}^{h t}$. Activation of the PUs degrade the connectivity of SUs. That is, for the connectivity of the secondary network, the spectrum holes unoccupied by the licensed PUs are required. Therefore, an increase in the activation rate of the PUs decreases the number of connected nodes in the secondary network, which eventually decreases the maximum total coverage area of the secondary subnetworks.

\section{CONCLUSiON}

For the WNCS applications requiring wide coverage areas, e.g., exploration and navigation, the maximum coverage area expression can be used for a cost-efficient multi-hop network ensuring the convergence of the estimator and hence the stability of the control system. Using the analysis presented in this technical note, the maximum total coverage area can be increased by appropriately adjusting the number of sensors, the successful transmission probability between nodes, the transmission range of nodes, and the eigenvalues of the system matrix.

\section{REFERENCES}

[1] Y. Halevi and A. Ray, "Integrated communication and control systems: Part I-Analysis," J. Dynamic Syst., Measure. Contr., vol. 110, pp. 367-373, Dec. 1988.

[2] M.S. Branicky, S.M. Phillips, and W. Zhang, "Stability of networked control systems: Explicit analysis of delay", in Proc. Amer. Control Conf., Chicago, IL, June 2000, pp. 2352-2357.

[3] A. Bemporad, M. Johansson, and M. Heemels, Networked Control Systems. Berlin, Germany: Springer, 2010

[4] D. Kilinc, M. Ozger, and O. B. Akan, "On the Maximum Coverage Area of Wireless Networked Control Systems under Stability and CostEfficiency Constraints," in Proc. IEEE GLOBECOM 2013, Atlanta, GA, USA, December 2013, pp. 274-279.

[5] C. F. Huang and Y. C. Tseng, "The coverage problem in a wireless sensor network," in Proc. ACM Int. Conf. Wireless Sensor Networks and Applications (WSNA), 2003, pp. 115-121.

[6] C. C. Tseng and K. C. Chen, "Power Efficient Topology Control in Wireless Ad Hoc Networks," in Proc. IEEE Wireless Comm. and Networking Conf. (WCNC), 2004, pp. 610-615.

[7] A. Utani, S. Nakagawa, and H. Yamamoto, "A Novel Data Gathering Scheme for Monitoring-Oriented Wireless Sensor Networks," International Journal of Innovative Computing, Information and Control, vol. 9, no. 1, pp. 111-122, 2013.

[8] O. B. Akan, O. Karli, and O. Ergul, "Cognitive radio sensor networks," IEEE Network, vol. 23, no. 4, pp. 34-40, Jul. 2009.

[9] I. F. Akyildiz, W. Y. Lee, and K. Chowdhury, "CRAHNs: Cognitive radio ad hoc networks," Ad Hoc Networks (Elsevier), vol. 7, no. 2, pp. 810-836, 2009.
[10] T. Fortmann, Y. Bar-Shalom, M. Scheffe, and S. Gelfand, "Detection thresholds for tracking in clutter - a connection between estimation and signal processing," IEEE Trans. Automat. Contr., vol. AC-30, pp. 221228, Mar. 1985.

[11] B. Sinopoli, L. Schenato, M. Franceschetti, K. Poola, M. I. Jordan, and S. S. Sastry, "Kalman filtering with intermittent observations", IEEE Trans. Autom. Control, vol. 49, no. 9, pp. 1453-1464, Sep. 2004.

[12] X. Liu and A. Goldsmith, "Kalman filtering with partial observation losses", in Proc. IEEE Conf. Decision and Control, Bahamas, vol. 4, pp. 4180-4186, Dec. 2004.

[13] R. Yang, P. Shi, and G. P. Liu, "Filtering for Discrete-Time Networked Nonlinear Systems With Mixed Random Delays and Packet Dropouts," IEEE Transactions on Automatic Control, vol. 56, no. 11, pp. 2655-2660, Nov. 2011.

[14] X. Su, L. Wu, and P. Shi, "Sensor Networks With Random Link Failures: Distributed Filtering for T-S Fuzzy Systems," IEEE Transactions on Industrial Informatics, vol. 9, no. 3, pp. 1739-1750, Aug. 2013.

[15] P. Wang, I. F. Akyildiz, and A. M. Al-Dhelaan, "Dynamic connectivity of cognitive radio ad-hoc networks with time-varying spectral activity," in Proc. IEEE GLOBECOM 2010.

[16] B. Karp and H. T. Kung, "GPSR: Greedy Perimeter Stateless Routing for Wireless Sensor Networks," in Proc. MobiCom 2000, Boston, MA, Aug. 2000.

[17] M. K. H. Fan and B. Nekooie, "On minimizing the largest eigenvalue of a symmetric matrix," Linear Algebra and its Applications, vol. 214, pp. 225-246, 1995. 